\title{
Uncertainty Relations for Information Entropy in Wave Mechanics
}

\author{
Iwo Białynicki-Birula* \\ Institute of Theoretical Physics, Warsaw University, 00-681 Warsaw, Poland, \\ and Department of Physics, University of Pittsburgh, Pittsburgh, USA \\ Jerzy Mycielski \\ Institute of Theoretical Physics, Warsaw University, 00-681 Warsaw, Poland
}

Received May 10, 1975

\begin{abstract}
New uncertainty relations in quantum mechanics are derived. They express restrictions imposed by quantum theory on probability distributions of canonically conjugate variables in terms of corresponding information entropies. The Heisenberg uncertainty relation follows from those inequalities and so does the Gross-Nelson inequality.
\end{abstract}

The purpose of this paper is to derive a new, stronger version of the Heisenberg uncertainty relation in wave mechanics. This new uncertainty relation has a simple interpretation in terms of information theory. It is also closely related to newly discovered logarithmic Sobolev inequalities.

The new uncertainty relation has the form (for wave functions normalized to unity)

$$
-\langle\ln \varrho\rangle-\langle\ln \tilde{\varrho}\rangle \geqq n(1+\ln \pi),
$$

where $\varrho$ and $\varrho$ are probability densities in $n$-dimensional position space and momentum space (or more precisely in wave-vector space),

$$
\begin{aligned}
& \varrho(\boldsymbol{r})=|\Psi(\boldsymbol{r})|^{2}, \\
& \varrho(\boldsymbol{k})=|\tilde{\Psi}(\boldsymbol{k})|^{2} .
\end{aligned}
$$

Brackets \langle\rangle denote integration over the whole position space or momentum space with $\varrho$ or $\varrho$. For example

$$
\langle\ln \varrho\rangle=\int d^{n} r \varrho(\boldsymbol{r}) \ln \varrho(\boldsymbol{r}) .
$$

Our normalization of the Fourier transform is

$$
\tilde{\Psi}(\boldsymbol{k})=\frac{1}{(2 \pi)^{n / 2}} \int d^{n} r \exp (-i \boldsymbol{k} \cdot \boldsymbol{r}) \Psi(\boldsymbol{r}) .
$$

It is worth observing that the inequality (1) does not depend on the unit of length used in measuring $\varrho$ and $\varrho$.

Unlike the standard uncertainty relation, which expresses indeterminacy of positions and momenta in terms of the second moments of the corresponding

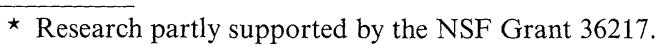


distributions, our uncertainty relation is expressed directly in terms of uncertainty measures used in information theory. Two integrals appearing on the 1.h.s. of (1) represent information theory entropies. The position-space entropy $-\langle\ln \varrho\rangle$ measures the uncertainty in the localization of the particle in space. The lower is this entropy the more concentrated is the wave function, the smaller is the uncertainty, and the higher is the accuracy in predicting the localization of the particle. In the same way momentum-space entropy $-\langle\ln \varrho\rangle$ measures the uncertainty in predicting the momentum of the particle. Each entropy taken separately decreases without bound when the corresponding probability density is becoming more and more concentrated, i.e. when the information is increasing. The boundedness from below of the sum of two entropies means that the total uncertainty in positions and in momenta can not be decreased beyond the value given in (1).

The inequality (1) can be derived from the recently ${ }^{1}$ obtained value of the $(p, q)$-norm of the Fourier transformation $[1,2]$. The $(p, q)$-norm of the Fourier transformation is the smallest number $k(p, q)$ for which the following inequality holds for all $\Psi \in L^{p}$

$$
\|\tilde{\Psi}\|_{q} \leqq k(p, q)\|\Psi\|_{p}
$$

where

$$
\begin{aligned}
& \|\Psi\|_{p}=\left(\int d^{n} r|\Psi|^{p}\right)^{1 / p}, \\
& \frac{1}{p}+\frac{1}{q}=1,
\end{aligned}
$$

and $q \geqq 2$. The calculated value of $k(p, q)$ is

$$
k(p, q)=\left(\frac{2 \pi}{q}\right)^{n / 2 q} \cdot\left(\frac{2 \pi}{p}\right)^{-n / 2 p} .
$$

Let us write now (2) in the form

$$
W(q) \equiv k(p, q)\|\Psi\|_{p}-\|\tilde{\Psi}\|_{q} \geqq 0,
$$

where $p$ is treated as a function of $q$ according to (3). Since $W(2)=0$ (ParsevalPlancherel theorem), the right derivative of $W(q)$ at $q=2$ must be nonnegative, i.e.

$$
\begin{aligned}
\frac{n}{4} N(1+ & \ln \pi)-\frac{1}{2} N^{-1} \int d^{n} r|\Psi(\boldsymbol{r})|^{2} \ln |\Psi(\boldsymbol{r})| \\
& \quad-\frac{1}{2} N^{-1} \int d^{n} k|\tilde{\Psi}(\boldsymbol{k})|^{2} \ln |\tilde{\Psi}(\boldsymbol{k})|+N \ln N \geqq 0,
\end{aligned}
$$

where $N=\|\Psi\|_{2}=\|\tilde{\Psi}\|_{2}$. For $N=1$, this inequality reduces to (1).

In the introduction we wrote that inequality (1) is stronger than the Heisenberg uncertainty relation. In order to prove this assertion we will first calculate the maximum value of the position-space entropy subject to two conditions

$$
\langle 1\rangle=1, \quad\left\langle(\boldsymbol{r}-\langle\boldsymbol{r}\rangle)^{2}\right\rangle=r_{0}^{2} .
$$

This problem is equivalent to finding the extremum of the following functional of $\varrho$

$$
-\langle\ln \varrho\rangle-\lambda(\langle 1\rangle-1)-\mu\left(\left\langle(\boldsymbol{r}-\langle\boldsymbol{r}\rangle)^{2}\right\rangle-r_{0}^{2}\right),
$$

1 The earlier result obtained in Ref. [1] is not sufficient for our purpose because it is restricted to integer values of $q$. 
where $\lambda$ and $\mu$ are Lagrange multipliers. By equating to zero the variation of this functional with respect to $\varrho$ we obtain

$$
\ln \varrho(\boldsymbol{r})+1+\lambda+\mu \boldsymbol{r}^{2}-2 \mu\langle\boldsymbol{r}\rangle \cdot \boldsymbol{r}=0 .
$$

The extremum determined by this condition is a true maximum since the entropy is a strictly concave functional of $\varrho$. The Lagrange multipliers $\lambda$ and $\mu$ can be determined with the use of the two subsidiary conditions (5) leading to the following results

$$
\begin{aligned}
\varrho_{\max }(\boldsymbol{r})= & \left(r_{0} \sqrt{2 \pi / n}\right)^{-n} \exp \left[-n(\boldsymbol{r}-\langle\boldsymbol{r}\rangle)^{2} / 2 r_{0}^{2}\right], \\
& -\langle\ln \varrho\rangle_{\max }=\frac{n}{2} \ln \left(2 \pi e r_{0}^{2} / n\right) .
\end{aligned}
$$

We have thus proven the inequality

$$
-\langle\ln \varrho\rangle \leqq \frac{n}{2} \ln \left(2 \pi e r_{0}^{2} / n\right),
$$

which can also be written in the form

$$
e \pi \exp \left(\frac{2}{n}\langle\ln \varrho\rangle\right) \geqq \frac{n}{2}\left(\left\langle(\boldsymbol{r}-\langle\boldsymbol{r}\rangle)^{2}\right\rangle\right)^{-1} .
$$

Owing to complete symmetry between the $r$ and $k$ spaces, we can write the same inequality for momentum-space entropy and dispersion in the $k$ variable. We choose to write it in the inverted form.

$$
\frac{2}{n}\left\langle(\boldsymbol{k}-\langle\boldsymbol{k}\rangle)^{2}\right\rangle \geqq(e \pi)^{-1} \exp \left(-\frac{2}{n}\langle\ln \varrho\rangle\right) .
$$

Our inequality (1) tells us, however, that the 1.h.s. of (6) does not exceed the r.h.s. of (7). To summarize our results we shall write the following chain of inequalities.

$$
\begin{aligned}
\frac{2}{n}\left\langle(\boldsymbol{k}-\langle\boldsymbol{k}\rangle)^{2}\right\rangle & \geqq(e \pi)^{-1} \exp \left(-\frac{2}{n}\langle\ln \varrho\rangle\right) \\
& \geqq e \pi \exp \left(\frac{2}{n}\langle\ln \varrho\rangle\right) \geqq \frac{n}{2}\left(\left\langle(\boldsymbol{r}-\langle\boldsymbol{r}\rangle)^{2}\right\rangle\right)^{-1}
\end{aligned}
$$

After the identification $\boldsymbol{p}=\hbar \boldsymbol{k}$, the first and the last part of this chain give the Heisenberg uncertainty in $n$ dimensions.

One can verify that all three inequalities in (8) become exact equalities for every Gaussian wave function. Moreover, it follows from our variational calculation that the first and the third inequalities become equalities only for Gaussian functions. We conjecture that this is also the case for the second inequality.

If we take only the first and the third term in (8) we obtain the Gross-Nelson (logarithmic Sobolev) inequality proved recently by Gross [3] and also studied by us in connection with wave equations with logarithmic nonlinearities ${ }^{2}$. To

\footnotetext{
${ }^{2}$ Actually, we arrived at inequality (1) while studying those equations.
} 
show this we may use an elementary inequality $e^{-1} e^{x} \geqq x$ to obtain from (8)

$$
\frac{2}{n}\left\langle(\boldsymbol{k}-\langle\boldsymbol{k}\rangle)^{2}\right\rangle \geqq \frac{2}{n}\langle\ln \varrho\rangle+2+\ln \pi
$$

or equivalently

$$
\int d^{n} r\left[(\nabla \Psi)^{*} \cdot(\nabla \Psi)-\Psi^{*} \Psi \ln \Psi^{*} \Psi\right] \geqq(\langle\boldsymbol{k}\rangle)^{2}+n(1+\ln \sqrt{\pi})
$$

as used by us in [4].

To end we shall derive an analog of inequality (1) for another pair of canonically conjugate variables, viz. for the angular momentum $M_{z}$ and the angle $\varphi$. This inequality is of the form

$$
-\sum_{m=-\infty}^{+\infty}\left|c_{m}\right|^{2} \ln \left|c_{m}\right|^{2}-\int_{0}^{2 \pi} \frac{d \varphi}{2 \pi}|\Phi(\varphi)|^{2} \ln |\Phi(\varphi)|^{2} \geqq 0,
$$

where $c_{m}$ are the Fourier coefficients in the expansion of a normalized wave function $\Phi(\varphi)$ into the series

$$
\Phi(\varphi)=\sum_{m=-\infty}^{\infty} c_{m} e^{i m \varphi} .
$$

Inequality (9) can be proved in the same manner as inequality (1) starting from the Hausdorff-Young inequality [5]

$$
\left(\sum_{m=-\infty}^{\infty}\left|c_{m}\right|^{q}\right)^{1 / q} \leqq\left(\int_{0}^{2 \pi} \frac{d \varphi}{2 \pi}|\Phi(\varphi)|^{p}\right)^{1 / p} .
$$

The sum of the two entropies in (9), measuring the total uncertainty in $M_{z}$ and $\varphi$ attains its lower bound if and only if the system is in an eigenstate of angular momentum.

Acknowledgments. One of the authors (IBB) would like to thank Professors J. Chadam and Z. Ciesielski for discussions.

\section{References}

1. Babenko, K. I.: Izv. Akad. Nauk SSSR. Ser. Mat. 25, 531 (1961)

2. Beckner, W.: Proc. Nat. Acad. Sci. U.S.A. 72, 638 (1975)

3. Gross, L.: Logarithmic Sobolev Inequalities (to appear in Amer. J. of Math)

4. Bialynicki-Birula,I., Mycielski, J.: Wave equations with logarithmic nonlinearities. Bull. Acad. Polon. Sci. Cl. III. 23, 461 (1975)

5. Zygmund, A.: Trigonometric series, Vol. II, p. 101. Cambridge University Press 1959

Communicated by R. Haag 